\title{
Rappels d'optique physique : interférences et diffraction
}

\author{
F. Reynaud et $P$. Bouchareine* \\ IRCOM, Université de Limoges, 123 rue Albert Thomas, 87060 Limoges cedex, France \\ * Institut d'Optique, B.P. 147, 91403 Orsay cedex, France
}

\begin{abstract}
Résumé : on étudie, dans le cadre de l'approximation de la représentation scalaire de la lumière, les phénomènes d'interférence à deux ondes, en rappelant les valeurs des paramètres principaux qui régissent ces phénomènes : interfrange, facteur de visibilité, influences des dimensions de la source, de son domaine spectral. Les interférences à ondes multiples sont vues dans les exemples du réseau et de l'interféromètre de Fabry et Perot. La diffraction par une pupille et sa représentation mathématique, le rôle de la transformation de Fourier en optique cohérente sont présentés dans la dernière partie.
\end{abstract}

\section{INTRODUCTION}

Les ondes lumineuses sont des ondes électromagnétiques qui oscillent à des fréquences extrêmement élevées. Dans le domaine visible, ces fréquences sont de l'ordre de $500 \mathrm{THz}$ (5 $10^{14} \mathrm{~Hz}$ ) conduisant, malgré leur très grande vitesse de propagation, à des longueurs d'onde inférieures au micromètre.

L'œil comme les détecteurs photoélectriques ne peuvent suivre les variations temporelles du champ électromagnétique. La réponse de ces détecteurs, qui représente un signal lumineux, est la valeur moyenne dans le temps du carré de l'amplitude du champ électromagnétique.

Dans ce contexte, la superposition en un même point de l'espace de deux ou plusieurs ondes monochromatiques de même fréquence et qui gardent une relation de phase constante sur une constante de temps du détecteur (ondes cohérentes) donne lieu à des phénomènes d'addition des amplitudes qui ne conduisent pas à des additions de flux, d'éclairements ou d'intensités.

Par exemple deux amplitudes égales qui s'ajoutent donnent une amplitude double, et un flux quadruple. Si les deux amplitudes se retranchent parce qu'elles sont en opposition de phase la résultante est nulle; comme le flux. Lorsqu'une amplitude 1 (unité arbitraire) tombe sur un détecteur en même temps qu'une amplitude 10, les deux flux sont dans le rapport 1 à 100. S'il y a cohérence entre les deux faisceaux, une interférence constructive conduit à une intensité $(10+1)^{2}=121$, et une interférence destructive donnera $(10-1)^{2}=81$. La différence est 40 fois plus forte que le seul flux de l'amplitude 1 . Les interférences donnent ici le principe d'une amplification très efficace des flux faibles par détection hétérodyne.

Dans la première partie nous étudierons les interférences à deux ondes à partir du dispositif historique des fentes d'Young. Dans la deuxième partie nous étudierons les interférences à ondes multiples avec le réseau et l'interféromètre de Fabry et Perot. Dans la troisième partie, nous étudierons la diffraction qui peut être présentée comme un phénomène 
d'interférence entre une infinité continue d'ondes d'amplitude infiniment petite. Les interférences se décrivent par des sommes algébriques ou vectorielles. La diffraction se décrit par des intégrales.

\section{INTERFÉRENCES À DEUX ONDES}

\subsection{Expériences des fentes d'Young}

\subsubsection{Dispositif expérimental}

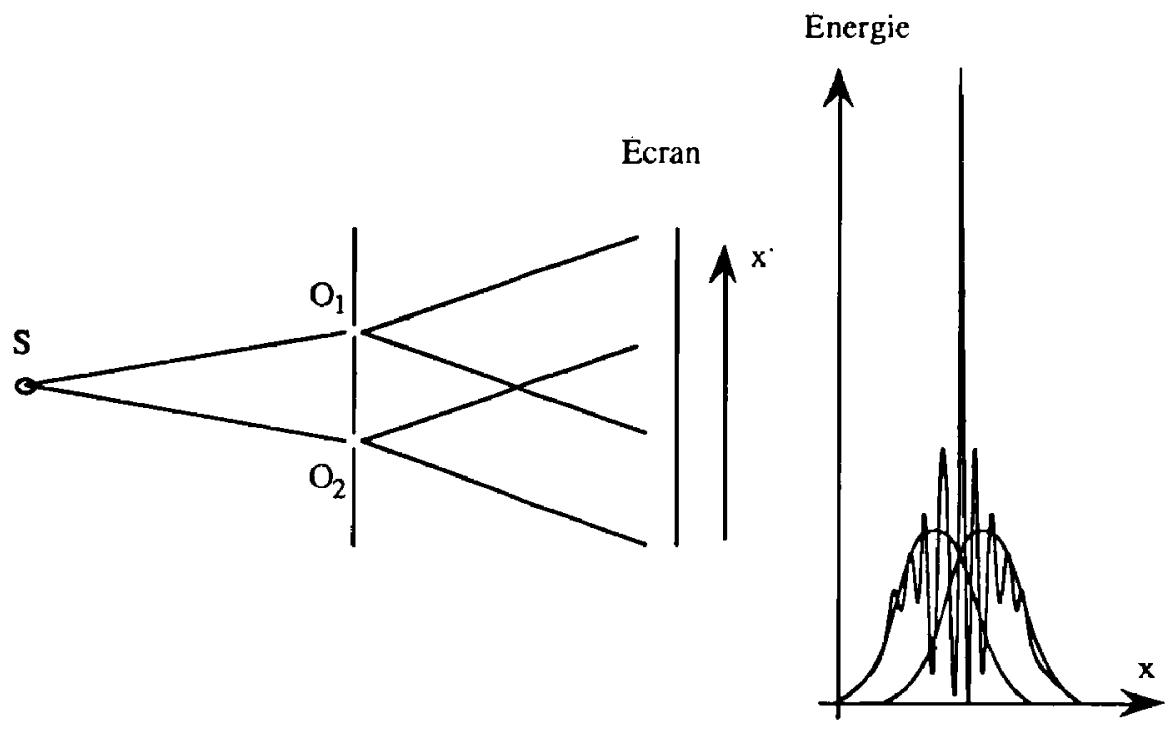

Figure 2.1 - Dispositif expérimental des franges d'Young.

Une source ponctuelle $S$ monochromatique (comme le problème est unidimensionnel il s'agit d'une fente fine perpendiculaire au plan de la figure 2.1) éclaire un écran percé de deux autres fentes fines $\mathrm{O}_{1}$ et $\mathrm{O}_{2}$. La lumière est diffractée par ces deux fentes de telle sorte que des rayons issus de ces deux fentes éclairent les mêmes zones du plan d'observation sur un écran. Dans la zone commune aux deux éclairements, on observe une modulation spatiale de la distribution d'énergie lumineuse sous forme de franges rectilignes équidistantes.

$\mathrm{Si}$ les fentes $\mathrm{O}_{1}$ et $\mathrm{O}_{2}$ sont très fines, on peut admettre un éclairement faible mais uniforme sur l'écran. C'est sur cette forme simplifiée que nous effectuerons les calculs. 


\subsubsection{Influence des divers parametres}

L'interfrange est plus grand avec de la lumière rouge qu'avec de la lumière bleue : il est proportionnel à la longueur d'onde. L'interfrange est proportionnel à la distance $\mathrm{D}$ entre l'écran d'observation et le plan des fentes. L'interfrange est proportionnel à l'inverse de la distance a entre les fentes diffractantes.

$$
\mathrm{i}=\lambda \mathrm{D} / \mathrm{a}
$$

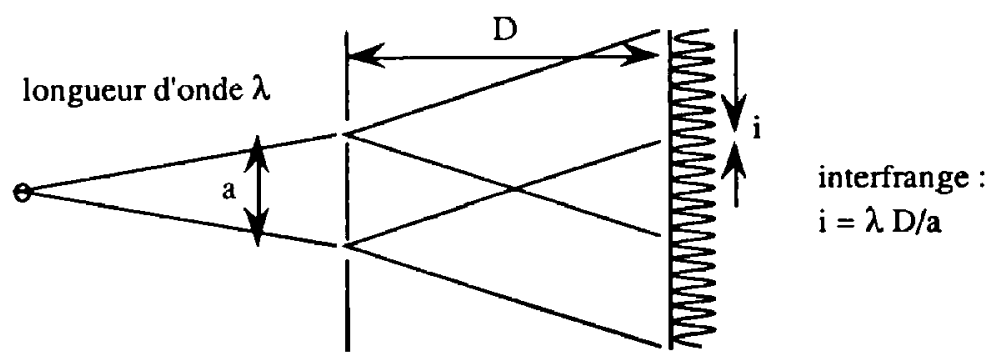

Figure 2..2 - Les paramètres du dispositif des franges d'Young.

\subsubsection{Différence de temps de propagation, différence de marche}

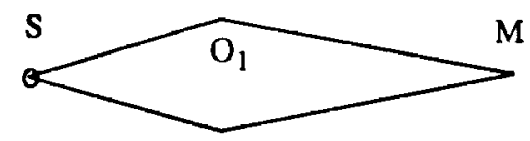

$\mathrm{O}_{2}$

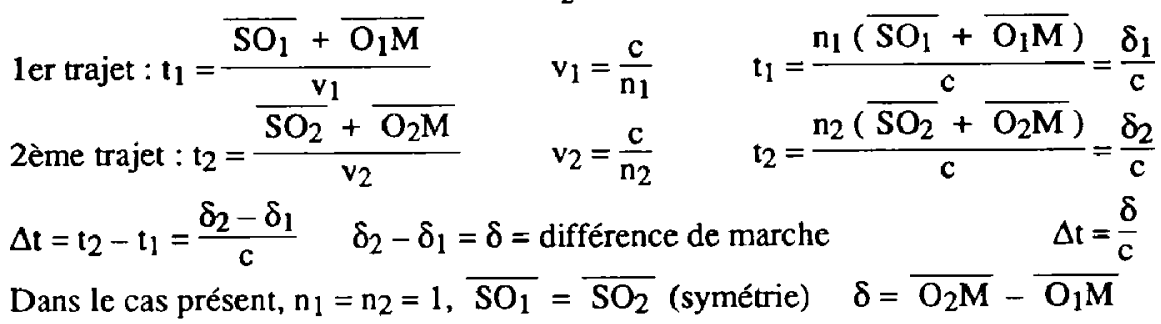

\subsection{Description mathématique des interférences}

\subsubsection{Superposition des champs}

Une onde électromagnétique monochromatique est la propagation synchrone d'un champ électrique et d'un champ magnétique qui varient sinusoïdalement avec le temps. La direction des champs caractérise la polarisation de l'onde. On simplifie la représentation d'une telle 
onde par un vecteur unique, le vecteur de Fresnel, dont l'expérience peut montrer qu'il est parallèle au champ électrique. Les solutions des équations de Maxwell déterminent les directions des champs électriques et magnétiques par rapport à la direction et au sens de propagation. Dans ces chapitres nous ne considérerons qu'un même état de polarisation pour toutes les ondes concernées, ce qui permet de travailler avec une approximation scalaire de l'onde électromagnétique, l'amplitude de l'onde égale au module du vecteur de Fresnel. L'élongation en un point fixe à chaque instant est égal au produit de cette amplitude par une fonction sinusoïdale du temps.

$$
E_{M}=E_{1}+E_{2}
$$

\subsubsection{Calcul de l'amplitude résultante pour une source monochromatique}

On rappelle que la constante de temps $\tau$ du détecteur est beaucoup plus longue que la période de l'oscillation du champ électromagnétique.

$$
\begin{gathered}
\text { Source }: E_{S}=a_{0} \cos (2 \pi v t) \\
E_{1}=a_{1} \cos \left(2 \pi v\left(t-t_{1}\right)\right) \\
E_{2}=a_{2} \cos \left(2 \pi v\left(t-t_{2}\right)\right)
\end{gathered}
$$

\subsection{Expression du signal détecté}

Le signal est proportionnel à ce que l'on appelle souvent l'intensité (c'est en réalité un flux ou un éclairement, c'est-à-dire un flux par unité de surface).

Le détecteur, que ce soit l'œil ou un détecteur photoélectrique, ne voit que la moyenne temporelle du carré des champs, qui est proportionnelle à l'éclairement.

\subsubsection{Remarque à propos d'une source unique}

$$
\begin{gathered}
E_{S}=a_{0} \cos \left(2 \pi v t+\varphi^{\prime}\right) \\
I=s=<E_{S}^{2}>_{t}=a_{0}^{2}<\cos ^{2}\left(2 \pi v t+\varphi^{\prime}\right)>t \\
I=\frac{a_{0}{ }^{2}}{2}
\end{gathered}
$$




\subsubsection{Application aux interférences}

$$
\begin{aligned}
& E_{M}=E_{1}+E_{2}=2 a \cos \left(2 \pi v t+\varphi^{\prime}\right) \cdot \cos (\pi \delta, \lambda) \\
& \mathrm{I}=\left\langle\mathrm{E}_{\mathrm{M}^{2}}>_{\mathrm{t}}=2 \mathrm{a}^{2} \cos ^{2}\left(\frac{\pi \delta}{\lambda}\right) \quad \cos ^{2}(\mathrm{x})=1 / 2(1+\cos (2 \mathrm{x}))\right. \\
& \text { Posant } \mathrm{I}_{\mathrm{O}}=\frac{\mathrm{a}^{2}}{2} \quad \mathrm{I}=2 \mathrm{I}_{\mathrm{o}}\left(1+\cos \left(\frac{2 \pi \delta}{\lambda}\right)\right)
\end{aligned}
$$

Note : on peut remplacer le calcul précédent par une construction géométrique, dite représentation de Fresnel (ne pas confondre avec le vecteur de Fresnel qui représente le caractère vectoriel des champs électromagnétiques). En effet, toute grandeur scalaire fonction sinusoïdale du temps peut être vue comme la composante sur un axe fixe d'un vecteur dont le module est l'amplitude de la modulation sinusoïdale et dont la phase à un instant donné est donnée par l'angle $\varphi$ entre ce vecteur et l'axe de référence. Plusieurs quantités synchrones seront représentées par des vecteurs tournant à la même vitesse angulaire, et formeront une figure géométrique fixe sur laquelle le calcul de la résultante peut être plus simple que le calcul analytique.

Dans le même ordre d'idées, on peut aussi faire ces calculs géométriques à partir des nombres complexes, chaque nombre permettant de représenter en module et en phase les vecteurs de la représentation de Fresnel. Nous utiliserons par la suite ces différents formalismes.

\subsubsection{Détermination du champ d'interférences dans l'expérience des fentes d'Young}

Le calcul se place dans le cadre des approximations précédentes. Il faut déterminer la différence de marche

$$
\begin{gathered}
\delta=\delta_{2}-\delta_{1}=\frac{a \cdot x}{D} \\
I=I_{0}\left(1+\cos \left(2 \pi \frac{a x}{\lambda D}\right)=2 I_{o}\left(1+\cos \left(2 \pi \frac{x}{\lambda D / a}\right)\right) \quad i=\lambda D / a\right. \text { est l'interfrange } \\
\begin{array}{ll}
\operatorname{maxima} 2 \pi x / i=2 k \pi \\
\operatorname{minima} x=(k+1 / 2) i
\end{array} \quad \begin{array}{l}
I_{\max }=4 I_{0} \\
I_{\min }=0
\end{array}
\end{gathered}
$$

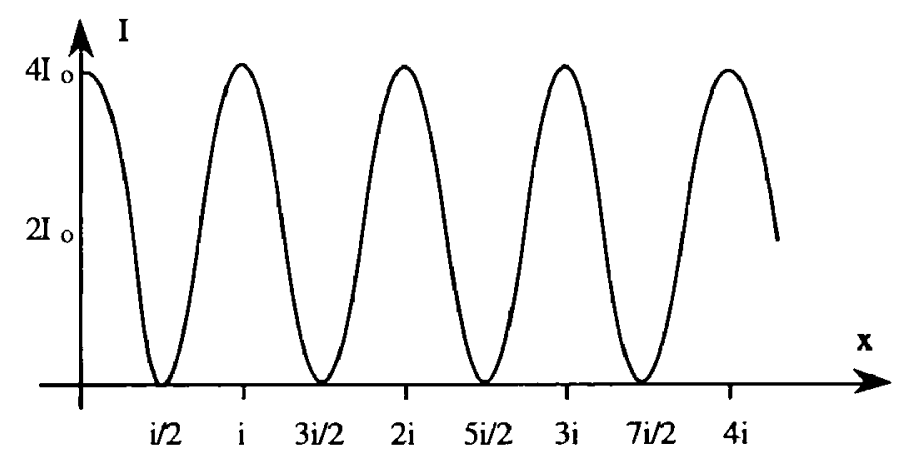

Figure 2.3 - Interférences à deux ondes. 


\subsubsection{Autres formulations}

Notion de différence de phase

$$
\mathrm{I}=2 \mathrm{I}_{0}(1+\cos \varphi) \quad \varphi=\varphi_{2}-\varphi_{1}=2 \pi \nu\left(\mathrm{t}_{2}-\mathrm{t}_{1}\right)=2 \pi \frac{\delta_{2}-\delta_{1}}{\lambda}
$$

Notion d'ordre d'interférence

$$
\mathrm{p}=\varphi / 2 \pi \quad \mathrm{I}=2 \mathrm{I}_{\mathrm{o}}(1+\cos (2 \pi \mathrm{p}))
$$

\subsection{Influence de la largeur spectrale de la source}

Dans une source non monochromatique, l'énergie électromagnétique est distribuée dans le domaine des fréquences (ou des longueurs d'onde) suivant une fonction que l'on appelle le spectre de la source.

Pour une différence de marche $\delta$ fixée, l'angle de phase $\varphi=2 \pi \frac{\delta}{\lambda}$ varie avec la longueur d'onde. Les interférences sont donc la superposition des états interférentiels des différentes radiations présentes dans le spectre de la source. On peut montrer que deux radiations de longueurs d'onde différentes ne peuvent interférer, ce sont donc les intensités résultant des interférences de chaque radiation qui s'ajoutent.

\subsubsection{Résultat sur les interférences avec une source polychromatique}

Une source monochromatique donne un signal sinusoïdal pour toutes les différences de marche.

Une source quasi monochromatique donne un signal sinusoïdal dont l'amplitude de modulation va en décroissant quand la différence de marche augmente. On caractérise cette modulation par le facteur de visibilité :

$$
\mathrm{V}=\frac{\mathrm{I}_{\max }-I_{\min }}{I_{\max }+I_{\min }} \quad \mathrm{V}_{\max }=1 \quad \mathrm{~V}_{\min }=0
$$

La différence de marche $\delta_{\max }$ à partir de laquelle le facteur de visibilité est très inférieur à l'unité est celle pour laquelle les variations de la phase avec les longueurs d'onde sont de l'ordre de $2 \pi$.

$$
\delta \varphi=2 \pi \delta / \lambda=2 \pi \sigma \delta
$$

La différence de marche $\delta_{\max }$ de visibilité des franges est à peu près égale à l'inverse de la largeur du spectre de la source exprimée en nombre d'ondes

$$
\delta_{\max }=\frac{1}{\Delta \sigma}
$$

Une source de lumière blanche (spectre large) donne une frange centrale brillante entourée de deux franges presque noires, puis rapidement l'intensité devient indépendante de la différence de marche $(\mathrm{V}=0)$; seules les couleurs varient avec cette différence de marche (échelle des teintes de Newton).

$$
\Delta \sigma=10000 \mathrm{~cm}^{-1} \quad \delta_{\max } \approx 1 \mu \mathrm{m}
$$


Interférence avec 2 radiations monochromatiques de fréquences voisines

La superposition des signaux interférentiels sinusoïdaux de périodes voisines donne lieu à un phénomène de battements. La période du battement en différence de marche est égale à la différence des nombres d'ondes des deux radiations. Avec un doublet dont les composantes sont d'intensités égales (doublet jaune du sodium, doublet jaune du mercure) la visibilité des franges s'annule périodiquement, permettant des pointés précis des anticoïncidences et une bonne mesure de l'intervalle spectral entre les deux composantes.

\subsubsection{Influence du temps de réponse du détecteur}

Si les composantes spectrales sont très proches et le temps de réponse du détecteur très court, on peut observer les interférences entre les deux composantes (battements entre deux lasers).

Radiations monochromatiques $v_{1}$ et $v_{2}$, pas d'interférences entre composantes sur de longues durées $\left(\tau\left(v_{2}-v_{1}\right)>1\right)$.

Temps de réponse court : $\tau\left(v_{2}-v_{1}\right)<1$. Le spectre de chaque composante est élargi $(\Delta v$ $\approx 1 / 2 \tau$ )

On observe les battements à la fréquence $\Delta v=v_{2}-v_{1}$.

\subsection{Influence de la largeur spatiale de la source}

\subsubsection{Notion de source étendue}

L'énergie électromagnétique dans la source peut être distribuée dans l'espace. La source est alors caractérisée par une luminance $L$ ou densité surfacique d'intensité. Si la différence de phase $\varphi$ entre les deux rayons dépend de la position du point source, la superposition des signaux interférentiels déphasés conduit à une perte du facteur de visibilité. En général le facteur de visibilité décroît quand les dimensions de la source augmentent.

Parfois la différence de phase reste constante, ou au moins stationnaire, quand les dimensions de la source augmentent. Ce phénomène n'est valable que dans un plan d'observation déterminé. On dit que les franges d'interférence y sont localisées. La localisation peut être parfaite, et le facteur de visibilité ne dépend pas des dimensions de la source (anneaux d'égales inclinaisons à l'infini) ; la localisation peut être approchée (cas des franges d'Young ou des franges de coin d'air).

Si la distribution des variations de chemin optique dans la source est uniformément distribuée sur un intervalle $\mathrm{d} \delta$, l'angle de phase $\varphi$ est uniformément distribué sur $\delta \varphi=2 \pi \mathrm{d} \delta / \lambda$ et le facteur de visibilité est donné par un sinus cardinal :

$$
\int_{\varphi}^{\varphi+\delta \varphi} 2 \mathrm{I}_{\circ}(1+\cos \varphi) d \varphi=2 \mathrm{I}_{\mathrm{o}}(1+\cos (\varphi+\delta \varphi / 2) \cdot \operatorname{sinc}(\delta \varphi / 2))
$$

Pour observer des franges avec un facteur de visibilité satisfaisant, on peut appliquer le critère de Lord Rayleigh : dans tout dispositif optique, on observe des phénomènes conformes au modèle utilisé si les défauts de chemin optique par rapport au modèle n'excèdent pas un quart de la longueur d'onde. Si l'on admet que le défaut est uniformément distribué sur un quart de longueur d'onde, l'angle de phase des interférences à deux ondes est uniformément distribué sur $\pi / 2$. La facteur de visibilité est alors égal à sinc $(\pi / 4)=0,90$. 


\section{INTERFÉRENCES À ONDES MULTIPLES}

\subsection{Réseau}

Reprenons l'expérience des fentes d'Young, mais au lieu de deux fentes, nous avons $\mathbf{N}$ fentes parallèles et équidistantes. Pour simplifier l'écriture nous supposons ces fentes très fines, c'est-à-dire que par la diffraction (voir le chapitre suivant), elles rayonnent uniformément dans l'espace. Nous supposerons aussi la fente source très fine et rejetée à l'infini dans la direction de la normale au plan des fentes diffractantes. On appelle un tel dispositif un réseau de diffraction, mais nous allons voir que l'essentiel des propriétés du champ électromagnétique provient des interférences à ondes multiples entre toutes ses fentes.

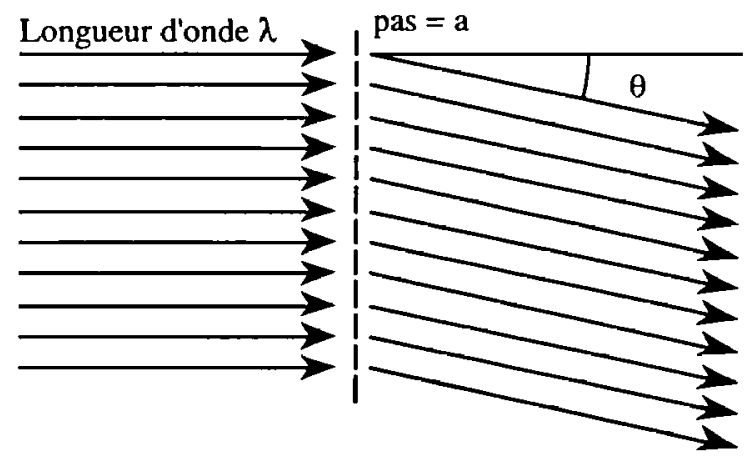

Figure 3.1 - Réseau à $\mathrm{N}$ fentes. Observation des interférences à l'infini.

$$
\delta=a \sin \theta \quad \varphi=a \sin (\theta) / \lambda .
$$

Nous observons l'onde électromagnétique à l'infini, dans une direction qui fait l'angle $\theta$ avec la normale au réseau. L'onde plane incidente éclaire les fentes avec une cohérence spatiale parfaite. La différence de phase entre deux sources secondaires représentées par deux fentes est parfaitement définie et égale à 0 (à cause de la direction de la source). Dans la direction caractérisée par l'angle $\theta$ la différence de phase entre deux fentes consécutives est

$$
\varphi=\frac{2 \pi \mathrm{a} \sin \theta}{\lambda}
$$

Dans la direction $\theta$ nous observons la superposition de $\mathrm{N}$ ondes d'amplitudes égales et dont les phases sont en progression arithmétique de période $\varphi$. La résultante s'écrit :

$$
\begin{gathered}
\left.E=E_{o}(1+\exp (-j \varphi)+\exp (-2 j \varphi)+\ldots+\exp (1-N) j \varphi)\right) \\
E \exp (-j \varphi)=E_{o}(\exp (-j \varphi)+\exp (-2 j \varphi)+\ldots+\exp (-N j \varphi)) \\
\text { d'où } E=E_{o} \frac{1-\exp (-N j \varphi)}{1-\exp (-j \varphi)}
\end{gathered}
$$


L'énergie correspondante est donnée par le carré du module de cette résultante qui s'écrit

$$
I=I_{o} / N^{2} \frac{\sin ^{2}(N \varphi / 2)}{\sin ^{2}(\varphi / 2)}
$$

La distribution des intensités est périodique de période $2 \pi$ en $\varphi$. On observe des maxima égaux dans la direction $\theta=0$ (ordre 0 ) et dans les directions telles que $\varphi=2 \mathrm{k} \pi$, $\mathrm{k}$ entier positif ou négatif est l'ordre d'interférence. Le signe de cet ordre est arbitraire et dépend de la convention de signe adoptée pour $\theta$.

L'ordre maximum est observé pour $\theta=\pi / 2$. Pour des directions voisines de $\theta=0$, les ordres sont angulairement équidistants :

$$
\Delta \theta=\lambda / \mathrm{a}
$$

Leur équidistance est égale au pas des franges d'Young données par deux fentes adjacentes.

Chaque pic est très semblable au carré d'un sinus cardinal. La largeur d'un pic est approximativement égale à la distance angulaire entre le sommet d'un pic et son premier zéro :

$$
\delta \theta=\lambda / \mathrm{Na}
$$

On peut caractériser ces ordres d'interférences par leur finesse $N=\Delta \theta / \delta \theta$ égale au nombre de fentes du réseau, c'est-à-dire au nombre d'ondes qui interfèrent.

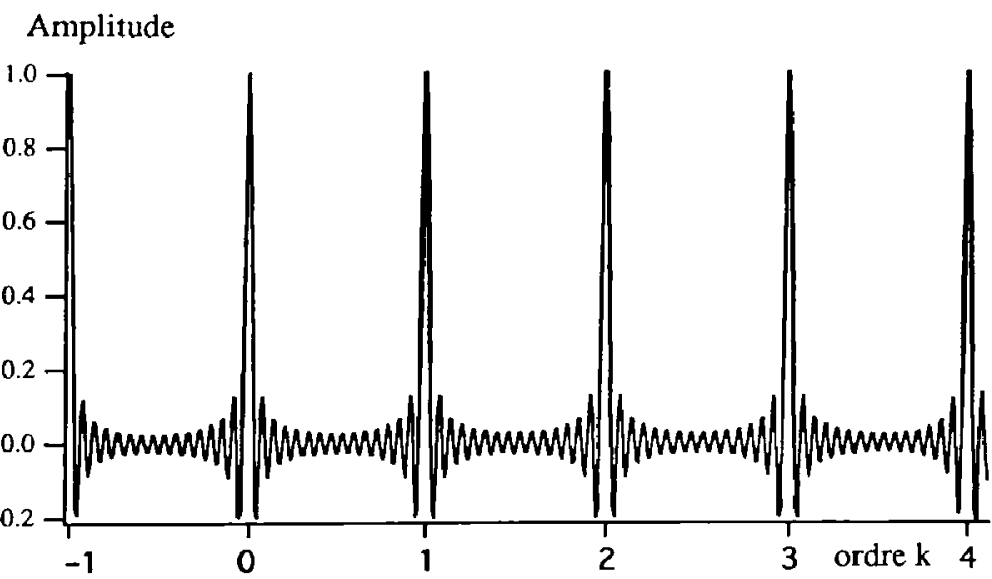

Figure 3.2 - Réseau : amplitude résultante à l'infini

Dans le cas général, l'angle d'incidence des ondes planes sur un réseau est $\theta_{1}$ et l'angle d'émergence est $\theta_{2}$. La condition d'observation de lumière monochromatique dans l'ordre $\mathbf{k}$ s'écrit : 
$a\left(\sin \theta_{1}+\sin \theta_{2}\right)=k \lambda$

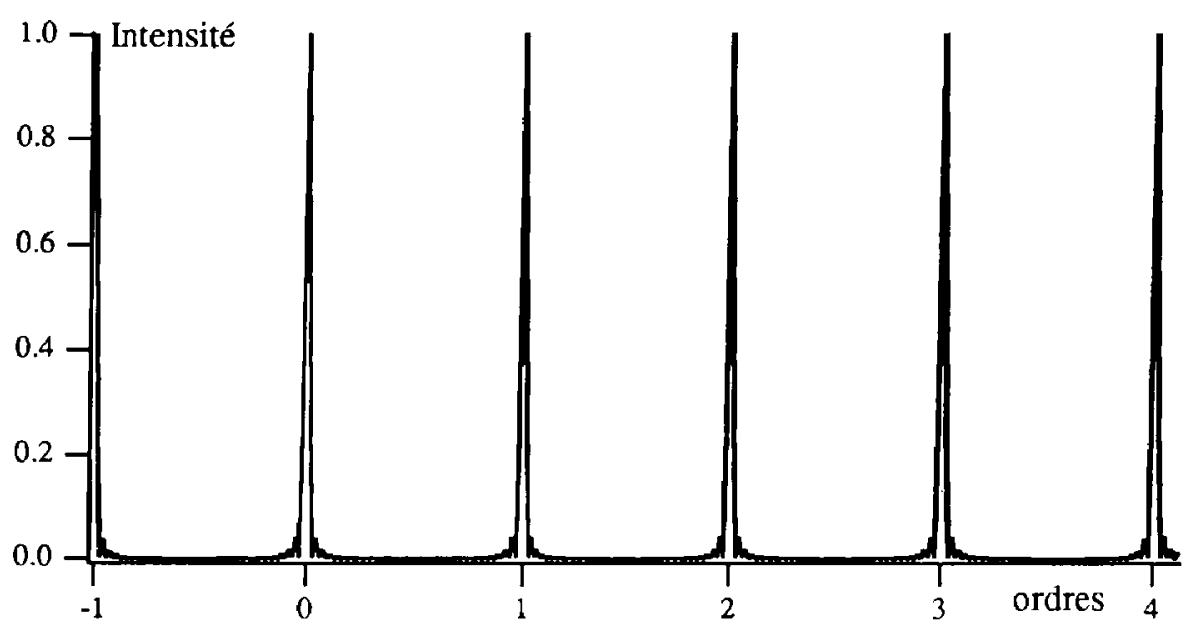

Figure 3.3 - Réseau : intensité à l'infuni.

\subsection{Formule d'Airy}

Deux miroirs plans parallèles se font face. Le facteur de réflexion de l'amplitude lumineuse de chaque miroir est $r$, le facteur de transmission est $t$, et le déphasage entre deux rayons dont l'un a subi deux réflexions entre les miroirs est :

$$
\varphi=2 \pi 2 n e \cos i / \lambda=4 \pi n e \sigma \cos i
$$

Tous les rayons issus d'un même rayon incident sous l'incidence i se retrouvent à l'infini, c'est-à-dire au foyer d'un objectif, et interferent en donnant l'amplitude résultante :

$$
E=E_{0} t^{2}\left(1+r^{2} \exp (j \varphi)+r^{4} \exp (-2 j \varphi)+\ldots+r^{2} N \exp (-N j \varphi)+\ldots\right)
$$

Le facteur de réflexion $r$ peut être complexe. Cela revient à ajouter un déphasage supplémentaire à $\varphi$ que l'on interprète souvent comme une épaisseur e modifiée, que l'on appelle l'épaisseur optique. Si $\delta \varphi$ est le déphasage à la réflexion éventuellement chromatique, l'épaisseur excédentaire $\delta \mathrm{e}$, variable avec la longueur d'onde, s'écrit :

$$
\delta \mathrm{e}=\lambda \delta \varphi / 2 \pi
$$

Nous admettrons qu'un éventuel déphasage, défini pour une radiation de longueur d'onde donnée, est inclus dans l'épaisseur optique e. 
Suivant John Biddel Airy, mathématicien anglais du XIXe siècle, nous écrivons la somme de cette suite en progression géométrique pour l'amplitude et en progression arithmétique pour la phase de la manière suivante :

$$
\begin{gathered}
E=E_{o} t^{2}\left(1+r^{2} \exp (j \varphi)+r^{4} \exp (-2 j \varphi)+\ldots+r^{2 N} \exp (-N j \varphi)+\ldots\right) \\
E r^{2} \exp (j \varphi)=E_{o} t^{2}\left(r^{2} \exp (j \varphi)+r^{4} \exp (-2 j \varphi)+\ldots+r^{2 N} \exp (-N j \varphi)+\ldots\right) \\
E\left(1-r^{2} \exp (j \varphi)\right)=E_{o} t^{2} \quad E=E_{0} \frac{t^{2}}{1-r^{2} \exp (j \varphi)}
\end{gathered}
$$

Nous obtenons l'expression de l'énergie lumineuse transmise en prenant le carré du module de $E$, soit en posant $r^{2}=R$ et $t^{2}=T$ :

$$
I=I_{0} \frac{T^{2}}{1-R^{2}} \frac{1}{1+\frac{4 R}{(1-R)^{2}} \sin ^{2} \varphi / 2}
$$

\subsection{Interprétation}

Cette fonction périodique en $\varphi$ de période $2 \pi$ a été présentée par J.B. Airy pour interpréter une petite différence entre la fonction sinusoïdale des interférences à deux ondes et la fonction observée dans l'expérience de Fizeau ou des anneaux de Newton entre les ondes réfléchies par deux lames de verre nu en vis-à-vis, avec un faible pouvoir réflecteur $R$.

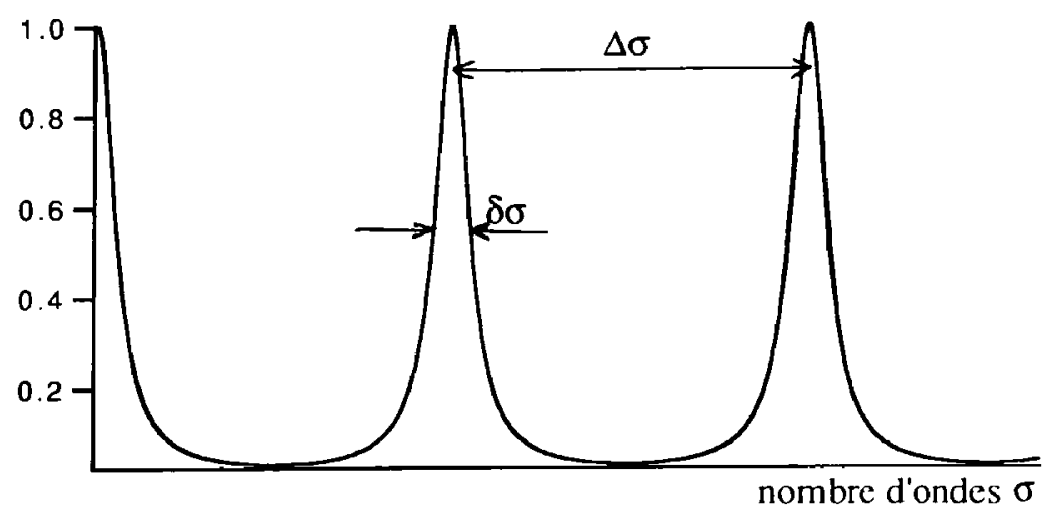

Figure 3.4 - Fonction d'Airy représentant les variations en fonction du nombre d'ondes de la radiation incidente du facteur de transmission d'un interféromètre de Fabry et Perot de finesse $9(R=0,71)$.

Cette périodicité en $\varphi$ de la fonction d'Airy peut être considérée sous trois points de vue différents :

En faisceau de rayons parallèles ( $\mathrm{i}=$ constante), l'interféromètre éclairé par un faisceau de lumiere blanche, c'est un facteur de transmission en fonction du nombre d'ondes. C'est le spectre cannelé transmis par l'interféromètre. 
Toujours en faisceau de rayons parallèles et en lumière monochromatique, c'est le facteur de transmission variable en fonction de l'épaisseur e.

En lumière monochromatique et en source étendue, c'est la transmission en fonction de l'angle d'incidence i qui donne une figure d'interférence caractéristique : les anneaux d'égales inclinaisons localisés à l'infini.

\subsection{Interféromètre de Fabry et Perot}

Il a fallu attendre les travaux de Charles Fabry et la soutenance de sa thèse en 1894 pour s'apercevoir que cette fonction prend des allures très particulières quand $R$ s'approche de l'unité. En effet le terme qui apparaît au dénominateur $4 R /\left(1-R^{2}\right)$ devient très grand, et la fonction de transmission devient une suite de résonances aiguës, qui n'a plus rien à voir avec une sinusoïde.

Charles Fabry a introduit la notion de finesse pour caractériser ces interférences comportant un très grand nombre d'ondes en progression géométrique décroissante : c'est le rapport entre la distance $\Delta \varphi=2 \pi$ entre deux pics et la largeur $2 \delta \varphi$ à mi-hauteur d'un pic.

$$
\mathrm{N}=\frac{2 \pi}{2 \delta \varphi}
$$

La finesse est un des rares termes de la langue française qui soit passé dans le langage scientifique international.

Le facteur de transmission égal à la moitié du maximum est obtenu pour

$$
\frac{4 \mathrm{R}}{(1-\mathrm{R})^{2}} \sin ^{2} \varphi=1
$$

Si $4 R /(1-R)^{2}$ est beaucoup plus grand que l'unité, la valeur de $\varphi$ correspondante est très proche de $2 \mathrm{k} \pi$, $\mathrm{k}$ étant un entier, et on peut remplacer $\sin (2 \mathrm{k} \pi+\delta \varphi) \operatorname{par} \delta \varphi$.

$$
\delta \varphi^{2}=\frac{(1-\mathrm{R})^{2}}{4 \mathrm{R}} \quad \mathrm{N}=\frac{2 \pi}{2 \delta \varphi}=\frac{\pi \sqrt{\mathrm{R}}}{1-\mathrm{R}}
$$

On peut vérifier sur cette expression que la quantité $\mathrm{R}^{2 \mathrm{~N}}$ est à peu près indépendante de $\mathrm{N}$ et vaut $\exp (-2 \pi)=1,910^{-3}$. C'est l'intensité relative par rapport au premier rayon transmis $\mathrm{du}(\mathrm{N}+1)^{\text {ème }}$ rayon. Cette constatation permet de donner un tout autre sens à la finesse : c'est le nombre de rayons à prendre en compte dans la suite infinie des amplitudes décroissantes. Les franges d'interférence de finesse $\mathrm{N}$ présentent à peu près la même largeur que des interférences à $\mathrm{N}$ ondes de même amplitude (voir le réseau dans le paragraphe précédent).

Charles Fabry ne disposait que d'argenture pour augmenter le pouvoir réflecteur des lames de verre. Avec des facteurs de réflexion de l'ordre de $75 \%$, il obtenait des finesses de l'ordre de 10. Aujourd'hui on sait faire des empilements de couches diélectriques dont le facteur de réflexion est aussi élevé que l'on souhaite. Des finesses de l'ordre de 50 à 100 sont courantes. Certaines cavités à très haut facteur de qualité peuvent offrir, pour une longueur d'onde bien définie, des finesses de plusieurs dizaines ou centaines de milliers (cavités pour gyrolasers, systèmes de stabilisation en fréquence de lasers pour la métrologie). 


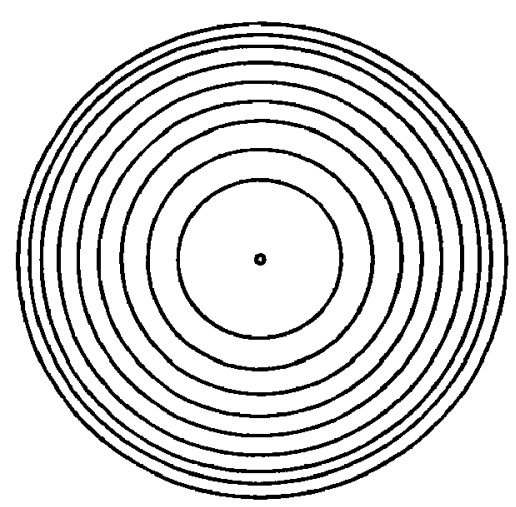

Figure 3.5 - Anneaux d'égales inclinaisons, localisés à l'infini, d'un interféromètre de Fabry et Perot éclairé par une source monochromatique étendue. Le centre des anneaux correspond à l'incidence normale sur les lames de

l'interféromètre. La structure des anneaux est la même que pour un interféromètre de Michelson, mais les anneaux sont fins au lieu d'avoir un profil sinusoïdal.

\subsection{Applications}

L'application la plus répandue de l'interféromètre de Fabry et Perot est la fabrication des filtres interférentiels, beaucoup plus sélectifs que les filtres colorés. Deux couches multidiélectriques à fort pouvoir réflecteur sont déposées de part et d'autre d'une lame de verre aux faces polies planes et parallèles. Ce Fabry-Perot solide est indéréglable, mais sa bande passante est variable avec l'angle d'incidence. Ce fait peut être exploité pour ajuster finement une bande passante vers une longueur d'onde un peu plus petite que celle du maximum de transmission en incidence normale.

On trouvera dans le cours "Spectrométrie instrumentale" une étude plus détaillée des propriétés spectrométriques de l'interféromètre de Fabry et Perot.

L'interféromètre de Fabry et Perot, rendu célèbre par la mesure du mètre en longueurs d'onde effectuée par Charles Fabry en 1907, fut très utilisé pour l'étude des spectres atomiques en haute résolution (structures hyperfines, déplacements isotopiques). Puis il fut oublié au profit d'autres méthodes interférentielles. Cet interféromètre est revenu en force avec le développement des lasers. C'est une cavité résonnante de type Fabry-Perot qui forme le cœur des lasers, et ce sont souvent des cavités résonnantes extérieures qui permettent d'asservir le laser en fréquence ou d'étudier les résonances atomiques ou moléculaires en très haute résolution.

\section{DIFFRACTION}

\subsection{Notion intuitive de la divergence d'un faisceau limité.}

Afin de comprendre l'origine du phénomène de diffraction observons la lumière émergeant d'un masque percé d'un trou de largeur $D$ éclairé par une onde plane monochromatique de longueur d'onde $\lambda$. L'observateur placé à grande distance voit arriver les contributions du champ ayant traversé la pupille en ses différents points. Afin de simplifier le raisonnement nous décomposerons la pupille en une suite discrète de zones. 


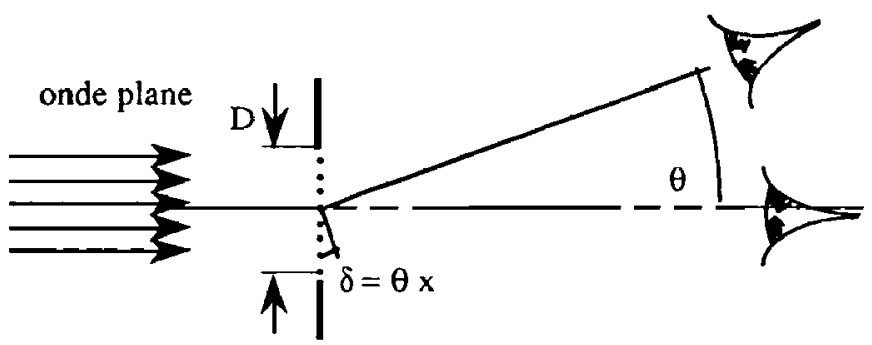

Figure 4.1 - Diffraction d'une onde plane par une pupille.

Additionnons les contributions en amplitude de chaque zone par une représentation de Fresnel. L'angle du vecteur par rapport au repère représente la phase du champ correspondant. Nous pouvons ainsi réaliser la superposition des champs parvenant à l'observateur par une méthode graphique.
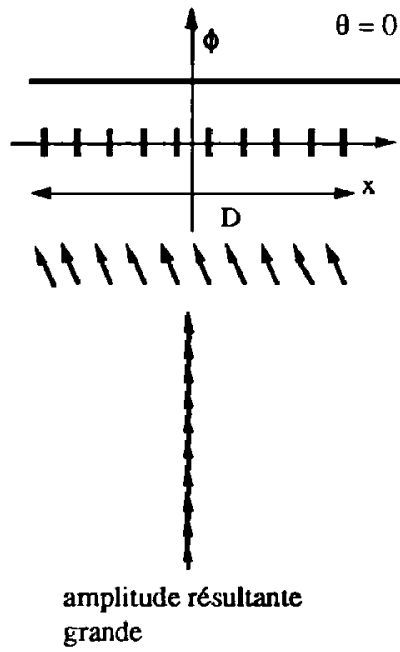
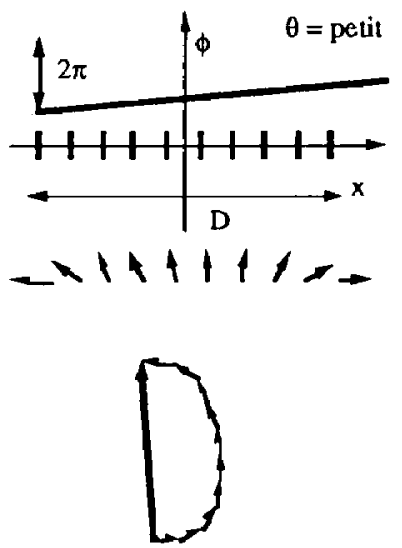

amplitude résultante moyenne
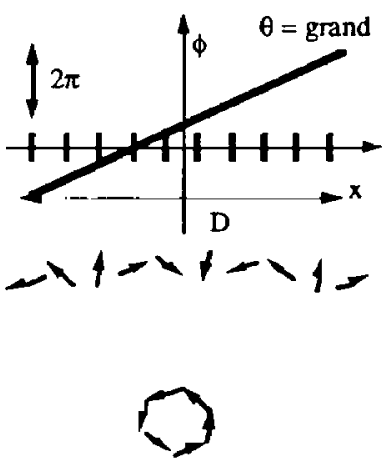

amplitude résultante \#0

Figure 4.2 - Diffraction par une pupille, construction géométrique de Fresnel.

Pour une observation sur l'axe l'ensemble des contributions ont la même phase. L'amplitude résultante est donc maximale. Observons maintenant la pupille diffractante sous un angle faible. Les différentes contributions sont faiblement déphasées L'amplitude résultante est plus faible que précédemment mais constitue une onde significative. Au contraire, dès lors que l'observateur incline sa direction de visée, le déphasage des divers contributions varie très vite conduisant à une résultante presque nulle.

Pour comprendre la notion de divergence d'un faisceau limité regardons à partir de quel seuil l'onde résultante commence à s'affaiblir significativement. Cette situation se rencontre dès que deux amplitudes élémentaires se superposent destructivement. Le seuil est donc atteint lorsque l'amplitude de variation du déphasage sur l'ensemble de la pupille est égal à $\pi$. 


$$
\varphi_{\text {seuil }}=\pi=\frac{2 \pi \cdot \delta_{\text {senil }}}{\lambda}=\frac{2 \pi \cdot \theta_{\text {seidit }} \cdot D}{\lambda}
$$

Le faisceau est donc contenu dans un cône de demi-angle au sommet

$$
\theta_{\text {seat }}=\frac{\lambda}{2 D}
$$

L'angle de divergence totale d'une onde plane limitée de diamètre $\mathrm{D}$ est donc $\lambda \mathrm{D}$.

Afin de préciser quantitativement les notions qualitatives exposées dans ce paragraphe nous allons maintenant proposer la décomposition d'une onde en ondes planes et appliquer cette technique pour calculer les figures de diffraction à distances finies ou infinies.

\subsection{Formalisme plus complet}

\subsubsection{Décomposition en ondes planes.}

Nous allons montrer comment utiliser les ondes planes pour analyser le phénomène de propagation pour une onde quelconque. Pour cela commençons par remarquer les deux points suivants :

* Une onde plane offre l'avantage de traduire le phénomène de propagation de façon très simple puisqu'il conduit à un simple déphasage du champ.

* Les ondes planes constituent une base de décomposition pour toute onde.

- Observons donc une onde plane d'expression générale :

$$
E(x, y, z)=E_{0} \exp (-j k . O M)
$$

où $\mathrm{x}, \mathrm{y}$ et $\mathrm{z}$ sont les coordonnées du vecteur position et $\mathrm{k}$ le vecteur d'onde de direction nornale aux surface d'onde et ayant pour valeur $k=2 \pi \frac{\mathrm{n}}{\lambda}$ si n est le vecteur unitaire dans cette direction qui sera choisie pour l'axe $\mathrm{Oz}$.

- Dans le plan $z=0$ le champ de cette onde a pour expression

$$
E(x, y, 0)=E_{0} \exp (-j k . O P)
$$

où $\mathrm{P}$ est un point du plan $(\mathrm{xOy})$. Cette expression peut s'écrire :

$$
E(x, y, 0)=E_{o} \exp \left(-\frac{2 \pi j}{\lambda}(\alpha x+\beta y)\right)
$$

si le vecteur $n$ a pour coordonnées $\alpha, \beta$ et $\gamma$. En introduisant un vecteur $N$ de coordonnées $N_{x}$, $N_{y}$ et $N_{z}$ égal à $n / \lambda$, le champ peut s'écrire :

$$
E(x, y, 0)=E_{0} \exp \left(-2 \pi j\left(x N_{x}+y N_{y}\right)\right) .
$$

Le vecteur $N$ apparaît donc comme la variable conjuguée du vecteur position $O P$ au sens de la transformée de Fourier. Ce vecteur est appelé vecteur fréquence spatiale. Notons que cette fonction élémentaire est un élément de la décomposition de Fourier à deux dimensions dans le plan (xOy). Ainsi tout champ peut être décomposé sous la forme : 


$$
E(x, y, 0)=\iint E_{0}\left(N_{x}, N_{y}\right) \exp \left(-2 \pi j\left(x N_{x}+y N_{y}\right)\right) d N_{x} \cdot d N_{y} .
$$

$\mathrm{E}_{\mathrm{o}}$ est appelé le spectre des fréquences spatiales. Il peut être calculé par l'expression suivante

$$
E_{0}\left(N_{x}, N_{y}\right)=\operatorname{TFE}(x, y, 0)=\iint E(x, y, 0) \exp \left(2 \pi j\left(x N_{x}+y N_{y}\right)\right) d x d y
$$

Pour traduire les phénomènes de propagation nous devons déterminer le déphasage introduit pour chaque onde plane servant dans la base de décomposition du champ. Ce calcul va être présenté à deux dimensions et peut être facilement généralisé au cas à trois dimensions.

Considérons une onde plane inclinée d'un angle $\theta$ par rapport à l'axe des $\mathbf{z}$.

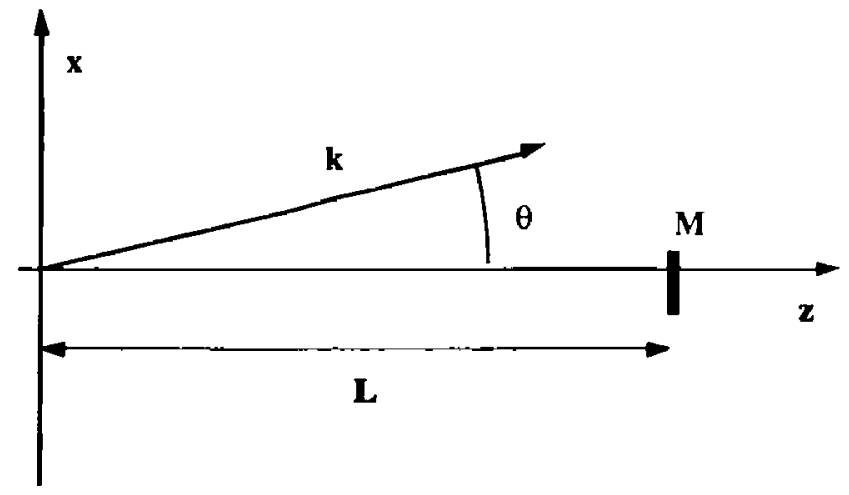

Figure 4.3

Son vecteur d'onde a pour expression $\mathrm{k}=2 \pi / \lambda(\cos \theta, \sin \theta)$. Dans le cas où $\theta$ reste petit (optique paraxiale) cette expression peut être approchée sous la forme

$$
k=2 \pi / \lambda\left(1,1-\theta^{2} / 2\right) \text {. }
$$

La fréquence spatiale correspondante est donc $\mathrm{N}_{\mathrm{x}}=\theta / \lambda$.

$\mathrm{D}$ 'autre part le déphasage introduit par une propagation suivant l'axe z sur une longueur L a pour expression :

$$
\varphi=k O M=2 \pi / \lambda L\left(1-\theta^{2} / 2\right)
$$

avec $\varphi=2 \pi \mathrm{L} / \lambda+\Delta \varphi$ où $\delta \varphi=-\pi \mathrm{L} \theta^{2} / \lambda$ traduisant la variation de la phase en fonction de la fréquence spatiale. Cette formule peut être généralisée au cas à trois dimensions sous la forme $: \varphi=2 \pi L / \lambda+\Delta \varphi$ avec $\Delta \varphi=\pi \mathrm{L}\left(\mathrm{N}_{\mathrm{x}}{ }^{2}+\mathrm{N}_{\mathrm{y}}{ }^{2}\right)$

Nous possédons maintenant tous les éléments pour décrire la propagation d'une onde sur une distance finie ou infinie. 
4.2.2 Diffraction de Fresnel ou diffraction à distance finie.

Afin de déterminer le champ diffracté à distance finie utilisons la méthode proposée sur le schéma 4.4 .

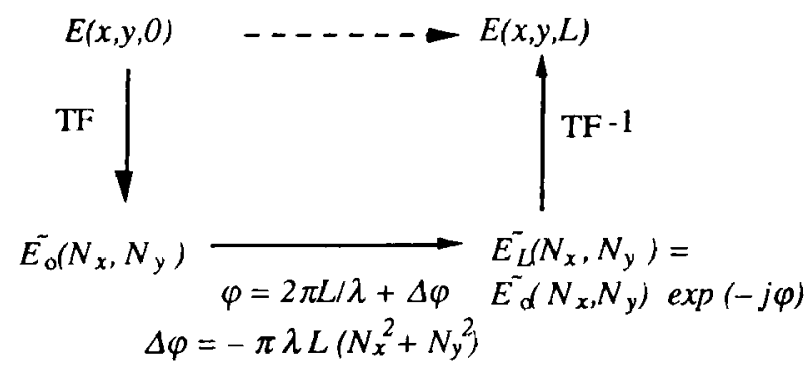

Figure 4.4

Le champ dans le plan de coordonnée $z=0$ est traité par transformation de Fourier afin de déterminer son spectre permettant la décomposition en ondes planes. Le phénomène de propagation est alors facile à exprimer en utilisant la méthode développée au paragraphe précédent. Un terme de phase $\varphi$ affecte les composantes du spectre suivant une loi parabolique en fonction des fréquences spatiales. L'expression du spectre de l'onde dans le plan $\mathrm{z}=\mathrm{L}$ permet, par transformation inverse, de calculer le champ dans ce même plan.

\subsubsection{Diffraction de Fraunhofer ou diffraction à distance infinie.}

Dans ce cas la distance $L$ tend vers l'infini. Le champ est constitué par les différentes ondes planes du spectre que la propagation a permis de séparer. La figure de diffraction est donc le spectre d'ondes planes lui même.

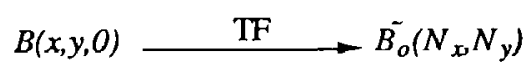

Figure 4.5

Notons que ce spectre est calculé en $\mathrm{z}=0$. Les termes de phase relative sont sans influence puisque les composantes du spectre sont séparées. L'intensité lumineuse s'obtient en prenant le module au carré du spectre. Dans ce cas les calculs sont plus simples et de nombreux exemples analytiques peuvent être proposés. Citons le cas de l'ouverture rectangulaire éclairée par une onde plane. En $z=0$ le champ s'exprime sous la forme :

$$
E(x, y, 0)=E_{o} \exp (-2 i \pi v t) \text { pour }-a / 2<x<a / 2 \text { et }-b / 2<y<b / 2
$$

Le spectre d'ondes planes associé a pour expression :

$$
E_{o}^{\prime}=a b \operatorname{sinc}\left(\pi a N_{x}\right) \cdot \operatorname{sinc}\left(\pi b N_{y}\right)
$$

L'intensité correspondante est : $I=I_{0} \operatorname{sinc}^{2}(\pi \mathrm{aN}) \cdot \operatorname{sinc}^{2}\left(\pi b N_{y}\right)$ 
Le même calcul effectué sur une pupille circulaire de rayon a donne la distribution d'intensité de la formule d'Airy qui met en cause une fonction de Bessel du premier ordre :

$$
I=I_{0}\left(\frac{2 J_{l}(\pi a \theta / \lambda)}{\pi a \theta / \lambda}\right)^{2}
$$

Les sections de ces deux distributions le long de l'axe des $x$ sont données par la figure 4.6.

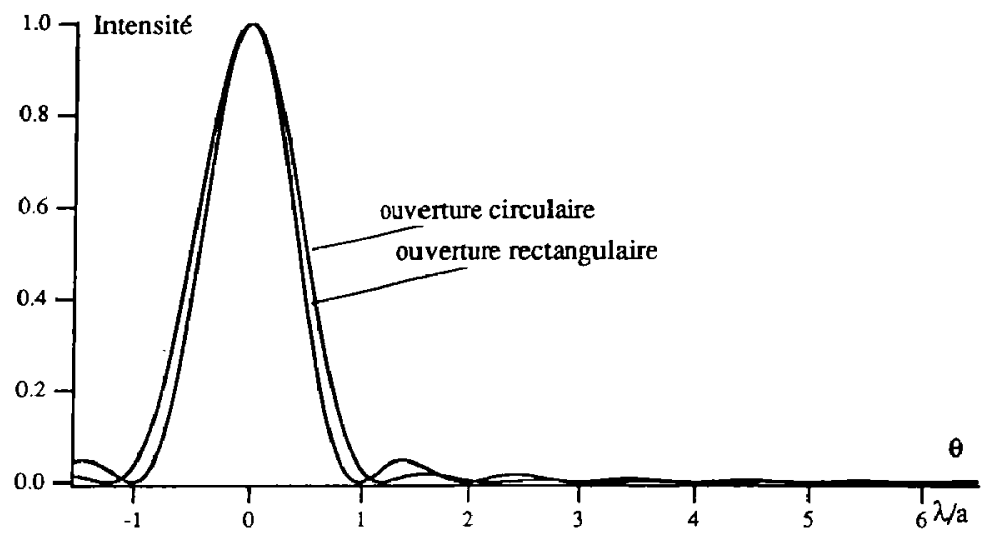

Figure 4.6 Figure de diffraction à l'infini d'une pupille rectangulaire de largeur a et d'une pupille circulaire de diamètre $\mathbf{a}$.

\subsubsection{Propagation des faisceaux gaussiens.}

Tout d'abord pourquoi s'intéresser à la propagation de faisceaux gaussiens ? La réponse se trouve dès que l'on commence à faire des calculs analytiques pour d'autres distributions. Les solutions analytiques simples ne sont pas légions et la fonction de Gauss offre le gros avantage de posséder une transformée de Fourier très simple à calculer, qui est encore une fonction de Gauss ; observons donc dans le plan $\mathrm{z}=0$ une onde de profil gaussien. Par souci de simplicité nous effectuerons le calcul pour une propagation à deux dimensions :

$$
E=E_{o} \exp \left(-\frac{\pi x}{a^{2}}\right)
$$

le spectre correspondant est $\mathscr{E}=\mathrm{a} \mathscr{E}_{0} \exp \left(-\pi \mathrm{a}^{2} \mathrm{~N}_{\mathrm{x}}{ }^{2}\right)$

Dans un plan de coordonnée $z=L$, le spectre des fréquences spatiales est modifié par le terme de phase résultant de la propagation:

$$
\tilde{E}_{L}=s . e^{-\pi N_{2}^{2} a^{2}} \cdot e^{-j \varphi} \text { avec } \varphi=\frac{2 \pi}{\lambda} \cdot L+\Delta \varphi \text { et } \Delta \varphi=-\pi \lambda L N_{x}^{2}
$$

La forme du faisceau correspondant est représentée sur la figure 4.7.

Un faisceau gaussien ayant une phase nulle en $\mathrm{z}=0$ possède trois domaines essentiels lors de sa propagation. Dans un premier temps le faisceau conserve une surface d'onde 
approximativement plane et un diamètre à peu près constant sur une longueur $L_{f}=a^{2} / \lambda$, appelée longueur de Fresnel. Cette propagation rectiligne correspond à la notion de rayon de l'optique géométrique.

Dans le deuxième domaine, après la longueur de Fresnel, le faisceau passe progressivement d'une forme cylindrique à une forme conique divergente. La surface d'onde se courbe progressivement. Cette phase est plus difficile à caractériser et correspond à un phénomène de diffraction dit de champ proche, sur une distance appelée longueur de Rayleigh $\mathrm{L}_{\mathrm{T}}=\pi \mathrm{a}^{2} / \lambda$.

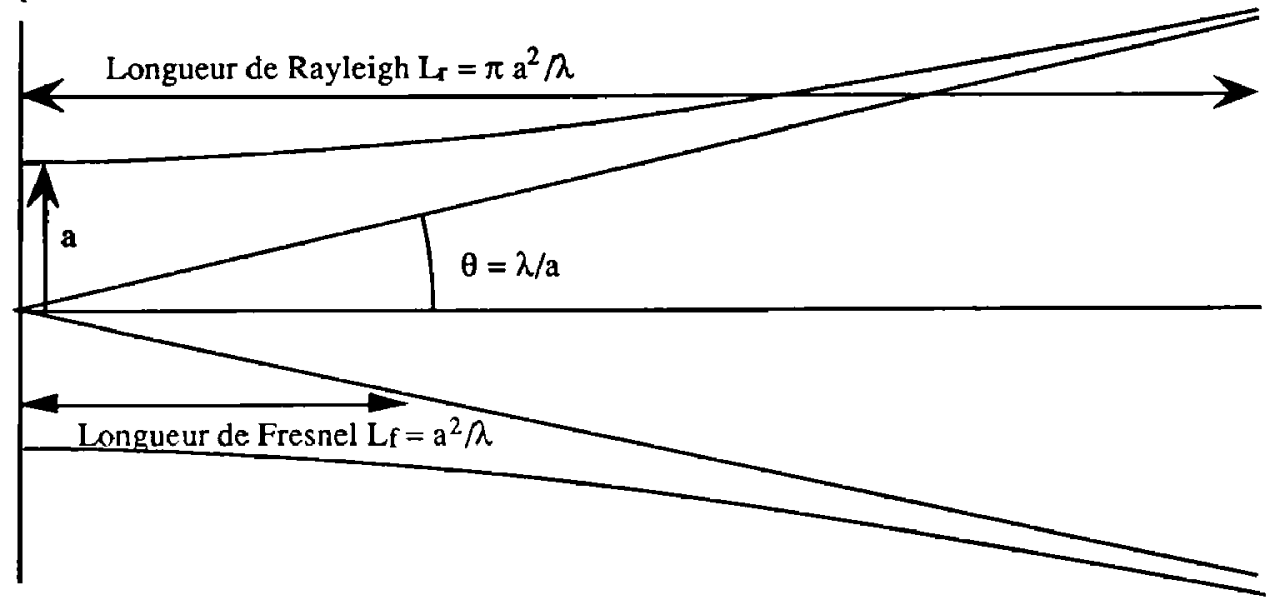

Figure 4.7 - Structure d'un faisceau gaussien.

Le troisième domaine intervient au delà de la longueur de Rayleigh. L'onde est alors sphérique et limitée, contenue dans un cône d'angle au sommet $\theta=\lambda / \mathrm{a}$. Cette zone est appelée champ lointain et possède les mêmes propriétés que la diffraction à l'infini. Le profil du faisceau évolue de façon homothétique en fonction de la distance de propagation.

Notons que l'étude réalisée dans le sens onde plane limitée $\rightarrow$ onde sphérique limitée fonctionne dans le sens inverse en appliquant le principe de retour inverse d'une onde. Le paragraphe suivant présente des applications de ces propriétés.

\subsection{A pplications}

\subsubsection{Liaison entre satellites par faisceau laser.}

La transmission d'un signal en utilisant comme support une onde est une forme tout à fait classique dans le domaine des ondes radio et micro-ondes. La transposition dans le domaine optique permettrait d'atteindre des débits d'information encore plus importants du fait de l'augmentation de la fréquence de l'onde porteuse. Malheureusement les hétérogénéités de l'atmosphère conduiraient, pour une observation au sol, à des rendements énergétiques absolument catastrophiques. Dans l'espace cette technique devient tout à fait realiste et a fait l'objet d'une étude afin de relier le satellite d'imagerie Spot avec des satellites géostationnaires afin de transmettre les images vers la terre de façon continue. Les antennes optiques sont constituées par des télescopes de diamètre $\mathrm{d}_{e m}$ émettant des ondes planes limitées que nous assimilerons à des faisceaux gaussiens (Figure 4.8). Les règles décrites précédemment permettent d'évaluer les rendements énergétiques lors de la transmission. Une 
approximation simple, considérant une répartition énergétique homogène des faisceaux sur des disques, conduit à un rendement de couplage $\rho$ entre la source et le récepteur égal au rapport de la surface collectrice du télescope de réception à la surface du faisceau au niveau du satellite.

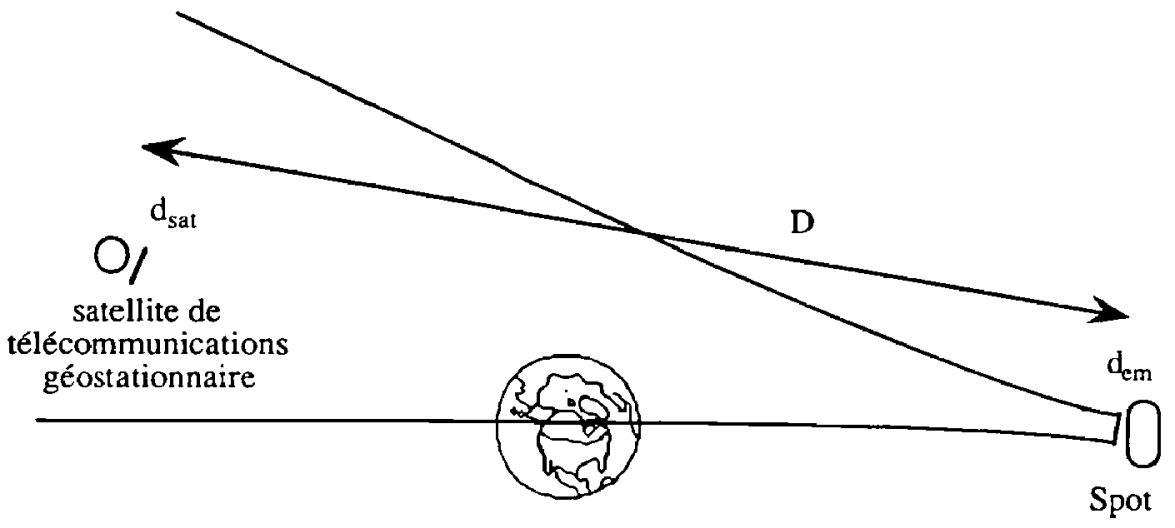

Figure 4.8 - Projet de liaison par faisceau laser entre le satellite Spot et un satellite de télécommunications géostationnaire.

$$
\rho=\frac{S_{\text {tel }}}{S_{\text {faisc }}}=\left(\frac{d_{\text {tel }}}{d_{\text {faisc }}}\right)^{2}
$$

Le rendement $\rho$ est donné en fonction de la distance $\mathrm{D}$ des télescopes par :

$$
\rho=\left(\frac{d_{\text {tel }}}{D \lambda / d_{e m}}\right)^{2}=\frac{d^{2}{ }_{t e l} d^{2} e m}{\lambda^{2} D^{2}}
$$

\subsubsection{Lecteur de disques optiques.}

Pour obtenir la meilleure résolution sur un disque optique, une lentille asphérique condense le faisceau du laser avec un angle d'ouverture voisin de $90^{\circ}$ qui donne au faisceau un diamètre de col de l'ordre de grandeur de la longueur d'onde. L'inconvénient d'un tel dispositif est sa faible profondeur de champ, qui est du même ordre de grandeur que la résolution latérale. C'est ce qui permet aux disques de la nouvelle génération d'enregistrer jusqu'à trois pistes différentes dans l'épaisseur du disque, pistes pouvant être lues successivement par le faisceau avec un ajustement en profondeur de la mise au point. 